\title{
RANK CHANGE ON ADJOINING REAL POWERS TO HARDY FIELDS
}

\author{
BY \\ MAXWELL ROSENLICHT ${ }^{1}$
}

\begin{abstract}
This paper concerns asymptotic approximations and expansions in cases where the usual Poincaré power series in $1 / x$ do not suffice because there may be more than one comparability class of functions that are very large or very small. The attempt to find asymptotic approximations in terms of real powers of given representatives of the comparability classes fails in general, but the situation can be saved by the adjunction of suitable real power products of the original functions, at the possible cost of an increase in the number of comparability classes.
\end{abstract}

1. Recall that a Hardy field is a set of germs of real-valued functions on positive half-lines which is closed under differentiation and which forms a field under addition and multiplication [1]. Examples of Hardy fields are $\mathbf{Q}, \mathbf{R}$, the field of rational functions $\mathbf{R}(x)$ (or rather the set of germs induced by these on suitable half-lines), and any extension field of a Hardy field obtained by adjoining a germ that is algebraic over the given Hardy field, or an antiderivative or the exponential of an element of the given Hardy field [1, Theorem 1 and Corollary 1 to Theorem 2].

Each element of a Hardy field approaches a limit in $\mathbf{R} \cup\{+\infty,-\infty\}$ as $x \rightarrow$ $+\infty$ and each nonzero element of a Hardy field ultimately has a constant sign. Nonconstant elements $f, g$ of a Hardy field $k$ that have the limits 0 or $\pm \infty$ as $x \rightarrow+\infty$ are called comparable if each of $|f|,|g|$ is bounded near $+\infty$, above and below, by integral powers of the other. The rank of $k$ is the number of comparability classes of such elements of $k[2, \S 3]$; for example, the Hardy field $\mathbf{R}\left(x, e^{x}\right)$ has rank 2 , with $x$ and $e^{x}$ representatives of its comparability classes.

Let $k$ be a Hardy field that contains $\mathbf{R}$ and let $t_{1}, t_{2}, \ldots, t_{n}$ be positive representatives of its various comparability classes. It often happens that if we adjoin to $k$ any set of real powers of $t_{1}, \ldots, t_{n}$ then the resulting Hardy field has the same rank $n$ as $k$, in which case for each nonzero $f \in k$ there are unique $c, c_{1}, \ldots, c_{n} \in \mathbf{R}$, with $c \neq 0$, such that $f \sim c t_{1}^{c_{1}} \cdots t_{n}^{c_{n}}$, and indeed each element of $k$ will have an asymptotic expansion in terms of such power products. But this happy circumstance, where the asymptotic expansions are so easy to manipulate, does not always occur. For example, consider the Hardy field

$$
k=\mathbf{R}\left(x, e^{x}, x^{a_{1}}, \ldots, x^{a_{r}}, \exp \left(b_{1} x+x^{a_{1}}\right), \ldots, \exp \left(b_{r} x+x^{a_{r}}\right)\right),
$$

where $a_{1}, \ldots, a_{r}, b_{1}, \ldots, b_{r} \in \mathbf{R}$, with $a_{1}, \ldots, a_{r}$ distinct numbers between 0 and 1 and $1, b_{1}, \ldots, b_{r}$ are linearly independent over $\mathbf{Q}$. Then $k$ has rank 2 , with $x$ and

Received by the editors August 20, 1983.

1980 Mathematics Subject Classification. Primary 34E05, 41A60; Secondary 12H05, 13N05, 26A12.

${ }^{1}$ Research supported by National Science Foundation grant number MCS-8303286 
$e^{x}$ representatives of its comparability classes, while

$$
k\left(e^{b_{1} x}, \ldots, e^{b_{r} x}\right)=\mathbf{R}\left(x, x^{a_{1}}, \ldots, x^{a_{r}}, e^{x}, e^{b_{1} x}, \ldots, e^{b_{r} x}, \exp \left(x^{a_{1}}\right), \ldots, \exp \left(x^{a_{r}}\right)\right)
$$

has rank $r+2$, with $x, e^{x}, \exp \left(x^{a_{1}}\right), \ldots, \exp \left(x^{a_{r}}\right)$ representatives of its comparability classes.

The main result of this paper is that if $k$ is a Hardy field containing $\mathbf{R}$ that is of finite rational rank (for example, if $k$ is obtained by adjoining to $\mathbf{R}$ a finite number of solutions of algebraic differential equations and all their derivatives) then there exists a Hardy field $K \supset k$, of rank at most the rational rank of $k$, such that any real power of any positive element of $K$ is in $K$ and, furthermore, a complete set of representatives of the comparability classes of $K$ may be found consisting of products of real powers of positive elements of $k$. This result follows immediately from Corollary 2 of the theorem in the next section. As a consequence, each element of $K$ has an asymptotic expansion in terms of constant multiples of real power products of any set of positive representatives of the various comparability classes of $K$.

2. We recall from [1 and 2] the canonical valuation of any Hardy field $k$, which is a homomorphism $\nu$ from the multiplicative group $k^{*}=k-\{0\}$ of $k$ onto an ordered abelian group (the value group) $\nu\left(k^{*}\right)$. The kernel of $\nu$ consists of all $f \in k^{*}$ such that $\lim _{x \rightarrow+\infty} f(x)$ is finite and nonzero, while $\nu(f)>0$ if and only if $\lim _{x \rightarrow+\infty} f(x)=0$. From [1 or 2], we know that if $f, g \in k^{*}$ and $\nu(f), \nu(g) \neq 0$, then $\nu(f) \geq \nu(g)$ if and only if $\nu\left(f^{\prime}\right) \geq \nu\left(g^{\prime}\right)$. From $[2, \S 1]$, we know that if $f, g \in k^{*}$, $\nu(f) \geq 0$ and $\nu(g) \neq 0$, then $\nu\left(f^{\prime}\right)>\nu\left(g^{\prime} / g\right)$, and the set $\left\{\nu\left(f^{\prime}\right): f \in k^{*}, \nu(f) \neq 0\right\}$ consists precisely of $\nu\left(k^{*}\right)$ minus the least upper bound (if this exists) in $\nu\left(k^{*}\right)$ of the set $\Psi(k)=\left\{\nu\left(u^{\prime} / u\right): u \in k^{*}, \nu(u) \neq 0\right\}$.

(This last point is not stated explicitly in $[2, \S 1]$, but is a direct consequence of Theorem 1 there, which states that if $f \in k^{*}$ and $\nu(f) \neq$ l.u.b. $\Psi(k)$ then for some $u \in k^{*}$ such that $\nu(u) \neq 0$ we have $f \sim\left(f\left(f u / u^{\prime}\right) /\left(f u / u^{\prime}\right)^{\prime}\right)^{\prime}$. We have only to show that $\nu\left(f\left(f u / u^{\prime}\right) /\left(f u / u^{\prime}\right)^{\prime}\right) \neq 0$. But otherwise $\nu(f)=\nu\left(\left(f u / u^{\prime}\right)^{\prime} /\left(f u / u^{\prime}\right)\right)$, which implies that $\nu(f) \in \Psi(k)$, unless $\nu\left(f u / u^{\prime}\right)=0$, or $\nu(f)=\nu\left(u^{\prime} / u\right) \in \Psi(k)$. Thus $\nu(f) \in \Psi(k)$, contrary to our knowledge that $\nu(f)$ exceeds each element of $\Psi(k)$.)

LEMMA 1. Let $k$ be a Hardy field, let $\Psi(k)=\left\{\nu\left(u^{\prime} / u\right): u \in k^{*}, \nu(u) \neq 0\right\}$, and let l.u.b. $\Psi(k)$ denote the least upper bound in $\nu\left(k^{*}\right)$ of $\Psi(k)$, if this exists. Then any element of $\nu\left(k^{*}\right)$ that exceeds l.u.b. $\Psi(k)$, if this exists, or otherwise exceeds each element of $\Psi(k)$, is of the form $\nu\left(f^{\prime}\right)$, for some $f \in k^{*}$ such that $\nu(f)>0$.

By the statement immediately preceding the lemma, each such element of $\nu\left(k^{*}\right)$ is of the form $\nu\left(f^{\prime}\right)$, for some $f \in k^{*}$ such that $\nu(f) \neq 0$. We must have $\nu(f)>0$, for otherwise $\nu\left(f^{\prime}\right)=\nu\left(f^{\prime} / f\right)+\nu(f)<\nu\left(f^{\prime} / f\right) \in \Psi(k)$.

LEMMA 2. Let $k \subset k(w)$ be Hardy fields, with either $w^{\prime} \in k$ or $w^{\prime} / w \in k$. Suppose that $w \sim 1$ and that there exists $t \in k^{*}$ such that $\nu(t)>0$ and $\nu\left(w^{\prime}\right) \geq \nu\left(t^{\prime}\right)$. Then $\nu\left((k(w))^{*}\right) \subset \mathbf{Q} \nu\left(k^{*}\right)$.

$\mathbf{Q} \nu\left(k^{*}\right)$ is the ordered vector space over $\mathbf{Q}$ spanned by $\nu\left(k^{*}\right)$, which by the first paragraph of $[2, \S 4]$ is the value group of the real algebraic closure of $k$. Since the real algebraic closure of $k(w)$ is a Hardy field, we may assume without 
loss of generality that $k$ is real algebraically closed. We may suppose $w$ to be transcendental over $k$. Then any nonzero element of $k(w)$ equals a nonzero element of $k$ times a product of positive or negative integral powers of elements of the form $w+a$, or $\left(w+a_{1}\right)^{2}+a_{2}^{2}$, with $a, a_{1}, a_{2} \in k, a_{2} \neq 0$. We therefore have to show that $\nu(w+a), \nu\left(\left(w+a_{1}\right)^{2}+a_{2}^{2}\right) \in \nu\left(k^{*}\right)$. We first examine $\nu\left(\left(w+a_{1}\right)^{2}+a_{2}^{2}\right)$, with $a_{1} \in$ $k, a_{2} \in k^{*}$. It is impossible to have $\nu\left(\left(w+a_{1}\right)^{2}+a_{2}^{2}\right) \neq \min \left\{\nu\left(\left(w+a_{1}\right)^{2}\right), \nu\left(a_{2}^{2}\right)\right\}$, for then $\left(w+a_{1}\right)^{2} \sim-a_{2}^{2}$, or $\left(\left(w+a_{1}\right) / a_{2}\right)^{2} \sim-1$. Thus

$$
\nu\left(\left(w+a_{1}\right)^{2}+a_{2}^{2}\right)=2 \min \left\{\nu\left(w+a_{1}\right), \nu\left(a_{2}\right)\right\}
$$

and it remains only to show that if $a \in k$ then $\nu(w+a) \in \nu\left(k^{*}\right)$. Since $w \sim 1$, we have $\nu(w+a)=\min \{0, \nu(a)\} \in \nu\left(k^{*}\right)$ unless $a \sim-1$. We may therefore assume $a \sim-1$, so that $\nu(w+a)>0$. Now let us suppose that $w^{\prime} \in k$. Let $g=w^{\prime}+a^{\prime} \in k$. We must have $g \neq 0$, for otherwise $w+a \in \mathbf{R}$, giving the false result $\nu(w+a)=0$, unless $w=-a$, contrary to the transcendence of $w$ over $k$. By the comments preceding Lemma $1, \nu(g) \geq \min \left\{\nu\left(w^{\prime}\right), \nu\left(a^{\prime}\right)\right\} \geq \min \left\{\nu\left(t^{\prime}\right), \nu\left(a^{\prime}\right)\right\}>$ l.u.b. $\Psi(k)$, if this exists, otherwise $\nu(g)$ exceeds each element of $\Psi(k)$. By Lemma 1 , there exists $f \in k^{*}$ such that $\nu(f)>0$ and $\nu\left(f^{\prime}\right)=\nu(g)$. Since $\nu(w+a), \nu(f) \neq 0$ and $\nu\left((w+a)^{\prime}\right)=\nu\left(f^{\prime}\right)$, we have $\nu(w+a)=\nu(f) \in \nu\left(k^{*}\right)$, completing the proof in the case $w^{\prime} \in k$. Finally consider the case $w^{\prime} / w \in k$. Here let $g=w^{\prime} / w-a^{\prime} / a \in k$. Then $g \neq 0$, for otherwise $(w / a)^{\prime}=0$, therefore $w / a \in \mathbf{R}$, so $(w+a) / a \in \mathbf{R}$, giving the false result $\nu(w+a)=\nu(a)=0$, unless $w=-a$, contrary to the transcendence of $w$ over $k$. Thus

$$
\begin{aligned}
\nu(g) & =\nu\left(w^{\prime} / w-a^{\prime} / a\right) \geq \min \left\{\nu\left(w^{\prime}\right), \nu\left(a^{\prime}\right)\right\} \\
& \geq \min \left\{\nu\left(t^{\prime}\right), \nu\left(a^{\prime}\right)\right\}>\text { l.u.b. } \Psi(k),
\end{aligned}
$$

if this exists, otherwise $\nu(g)$ exceeds each element of $\Psi(k)$. As above, there exists $f \in k^{*}$ such that $\nu(f)>0$ and $\nu\left(f^{\prime}\right)=\nu(g)$. Now $((w+a) / a)^{\prime}=(w / a)^{\prime}=$ $(w / a)\left(w^{\prime} / w-a^{\prime} / a\right)$. Thus $\nu(w+a), \nu(f) \neq 0$ and

$$
\nu\left((w+a)^{\prime}\right)=\nu\left(((w+a) / a)^{\prime}\right)=\nu\left(w^{\prime} / w-a^{\prime} / a\right)=\nu(g)=\nu\left(f^{\prime}\right),
$$

so that $\nu(w+a)=\nu(f) \in \nu\left(k^{*}\right)$, which completes the proof.

It would be interesting to be able to strengthen Lemma 2 by proving that $\nu\left((k(w))^{*}\right) \subset \nu\left(k^{*}\right)$.

The following Lemma 3 can be ignored by readers interested only in the case where the Hardy field $k$ has finite rank or, more generally, where $\max \Psi(k)$ exists. Indeed, the author has no examples of the cases described in this lemma, other than the trivial case $k \subset \mathbf{R}$. We make the prefatory remark that if $k \subset k(w)$ are Hardy fields and $w^{\prime} / w \in k$, then if $\nu\left(w^{\prime} / w\right)>$ l.u.b. $\Psi(k)$, if this exists, or if otherwise $\nu\left(w^{\prime} / w\right)$ is greater than each element of $\Psi(k)$, then $\nu(w)=0$, a consequence of Lemma 1 and the paragraph preceding it. However, if $k$ is any Hardy field, and $u, w \in k^{*}, \nu(u) \neq 0$ and $\nu\left(w^{\prime} / w\right) \leq \nu\left(u^{\prime} / u\right)$, then $\nu(w) \neq 0$ and the comparability class of $w$ is at least as large as that of $u$, with equality of these classes if and only if $\nu\left(w^{\prime} / w\right)=\nu\left(u^{\prime} / u\right)$, by [2, Propositions 3 and 4$]$.

LEMMA 3. Let $k \subset k(w)$ be Hardy fields, with $w^{\prime} / w \in k$ and $\nu\left(w^{\prime} / w\right) \notin \Psi(k)=$ $\left\{\nu\left(u^{\prime} / u\right): u \in k^{*}, \nu(u) \neq 0\right\}$, but $\nu\left(w^{\prime} / w\right)=$ l.u.b. $\Psi(k)$, the least upper bound being taken in $\nu\left(k^{*}\right)$. Then $\nu(w) \neq 0$ if and only if, for each $a \in k^{*}$ such that $\nu(a)=$ l.u.b. $\Psi(k)$ we have $\nu\left(\int a\right)<0$, that is an indefinite integral of $a$ is divergent. 
If $\nu(w) \neq 0$ then the comparability class of $w$ in $k(w)$ is minimal and we have $\nu\left((k(w))^{*}\right)=\nu\left(k^{*}\right)+\mathbf{Z} \nu(w), \Psi(k(w))=\Psi(k) \cup\left\{\nu\left(w^{\prime} / w\right)\right\}$, and $\max \Psi(k(w))=$ $\nu\left(w^{\prime} / w\right)=$ l.u.b. $\Psi(k)$. If $\nu(w)=0$, with $w \sim c \in \mathbf{R} \cap k^{*}$, and we set $v=w-c$, then $\nu(v)>0, \nu\left(v^{\prime}\right)=\nu\left(w^{\prime}\right)=$ l.u.b. $\Psi(k)$, the comparability class of $v$ in $k(w)$ is minimal, and we have

$$
\nu\left((k(w))^{*}\right)=\nu\left(k^{*}\right)+\mathbf{Z} \nu(v), \quad \Psi(k(w))=\Psi(k) \cup\left\{\nu\left(v^{\prime} / v\right)\right\}
$$

and

$$
\max \Psi(k(w))=\nu\left(v^{\prime} / v\right)=\text { l.u.b. } \Psi(k)-\nu(v) .
$$

If $a, b \in k^{*}$ and $a \sim b$, then each $a, b$ is ultimately positive or ultimately negative and the absolute value of each is bounded above and below by constant multiples of the absolute value of the other, so that $\int a$ diverges if and only if $\int b$ diverges. But $\int a$ diverges if and only if $\nu\left(\int a\right)<0$. Taking $a=w^{\prime} / w$, with $w$ as given above, we have $\int a=\log |w|$ and $\nu(\log |w|)<0$ if and only if $\log |w|$ is unbounded on any positive half-line, meaning that $w$ or $1 / w$ is unbounded on any positive half-line, or $\nu(w) \neq 0$. Now suppose we have the case $\nu(w) \neq 0$. Then for each $u \in k^{*}$ such that $\nu(u) \neq 0$ we have $\nu\left(w^{\prime} / w\right)>\nu\left(u^{\prime} / u\right)$, so that the comparability class of $w$ is smaller than that of $u$. Thus $\nu\left(f w^{i}\right) \neq \nu\left(g w^{j}\right)$ if $f, g \in k^{*}$ and $i, j$ are unequal integers, and it follows that $\nu\left((k(w))^{*}\right)=\nu\left(k^{*}\right)+\mathbf{Z} \nu(w)$. For $f, g \in(k(w))^{*}$ and $\nu(f)=\nu(g) \neq 0$ we have $\nu\left(f^{\prime} / f\right)=\nu\left(g^{\prime} / g\right)$, so that the statement about $\nu\left((k(w))^{*}\right)$ implies the various assertions about $\Psi(k(w))$. Suppose finally that $\nu(w)=0$ and $w \sim c \in \mathbf{R} \cap k^{*}$. If $v=w-c$, then $\nu(v)>0$ and $\nu\left(v^{\prime}\right)=\nu\left(w^{\prime}\right)=\nu\left(w^{\prime} / w\right)=$ l.u.b. $\Psi(k)>\nu\left(u^{\prime} / u\right)$ if $u \in k^{*}$ is such that $\nu(u) \neq 0$, and, as before, the comparability class of $v$ is smaller than that of $u$. Since $k(w)=$ $k(v)$ we deduce, exactly as above, that $\nu\left((k(w))^{*}\right)=\nu\left(k^{*}\right)+\mathbf{Z} \nu(v)$ and the various assertions about the present $\Psi(k(w))$ are immediate.

Let $k$ be a Hardy field containing $\mathbf{R}$. If $t$ is a positive (i.e. ultimately positive) element of $k$ and $c \in \mathbf{R}$, then $k\left(t^{c}\right)$ is a Hardy field. We call $t^{c}$ a real power of $t$. The expression $\nu\left(t^{c}\right)$ makes sense, as an element of the value group of $k\left(t^{c}\right)$, or indeed the value group of any extension Hardy field of $k\left(t^{c}\right)$. If $t_{1}, t_{2} \in k^{*}$ and $\nu\left(t_{1}\right)=\nu\left(t_{2}\right)$ then $t_{2} \sim a t_{1}$ for some $a \in \mathbf{R}^{*}$, so that $\left|t_{2}\right|^{c} \sim|a|^{c}\left|t_{1}\right|^{c}$ and therefore $\nu\left(\left|t_{2}\right|^{c}\right)=\nu\left(\left|t_{1}\right|^{c}\right)$. It therefore makes sense, for $\alpha \in \nu\left(k^{*}\right)$ and $c \in \mathbf{R}$, to define $c \alpha$ to be $\nu\left(|t|^{c}\right)$, where $t$ is any element of $k$ such that $\nu(t)=\alpha$. We deduce immediately that $c(\alpha+\beta)=c \alpha+c \beta$ for $c \in \mathbf{R}$ and $\alpha, \beta \in \nu\left(k^{*}\right)$, that $\left(c_{1}+c_{2}\right) \alpha=c_{1} \alpha+c_{2} \alpha$ and $c_{1}\left(c_{2} \alpha\right)=\left(c_{1} c_{2}\right) \alpha$ for $c_{1}, c_{2} \in \mathbf{R}$ and $\alpha \in \nu\left(k^{*}\right)$, and that $1 \alpha=\alpha$ for each $\alpha \in \nu\left(k^{*}\right)$. If $\alpha \in \nu\left(k^{*}\right)$ and $c \in \mathbf{Q}$, the present $c \alpha$ has the same sense as earlier, when $c \alpha \in \mathbf{Q} \nu\left(k^{*}\right)$. If $K$ is an extension Hardy field of $k$ that is closed under the taking of real powers of positive elements, then the value group $\nu\left(K^{*}\right)$ is a vector space over $\mathbf{R}$. A main object of study is the subspace $\sum_{t \in k^{*}} \mathbf{R} \nu(t)$, each element of which is of the form $\nu(u)$, for some real power product of elements of $k u=\left|t_{1}\right|^{c_{1}} \cdots\left|t_{n}\right|^{c_{n}}$, where each $t_{i} \in k^{*}$ and each $c_{i} \in \mathbf{R}$. Note that for such a $u$ we have $u^{\prime} / u=c_{1} t_{1}^{\prime} / t_{1}+\cdots+c_{n} t_{n}^{\prime} / t_{n} \in k$, so that for such a $u$ the field $k(u)$, as a subfield closed under differentiation of a Hardy field, is itself a Hardy field.

THEOREM. Let $k$ be a Hardy field that contains $\mathbf{R}$ and let

$$
\hat{k}=k\left(\left\{|t|^{c}: t \in k^{*}, c \in \mathbf{R}\right\}\right) \text {. }
$$

Write $\Psi(k)=\left\{\nu\left(u^{\prime} / u\right): u \in k^{*}, \nu(u) \neq 0\right\}$. Then $\hat{k}$ is a Hardy field and: 
(1) if $\max \Psi(k)$ exists, then $\nu\left(\hat{k}^{*}\right)$ is the $\mathbf{R}$-span of $\nu\left(k^{*}\right)$, that is $\nu\left(\hat{k}^{*}\right)=$ $\mathbf{R} \nu(k)^{*}=\sum_{t \in k^{*}} \mathbf{R} \nu(t)$, and $\max \Psi(\hat{k})=\max \Psi(k)$;

(2) if there is no real power product $w$ of elements of $k$ such that $\nu\left(w^{\prime} / w\right)$ is the least upper bound of $\Psi(k)$ in $\nu\left(k^{*}\right)$, then $\nu\left(\hat{k}^{*}\right)=\mathbf{R} \nu\left(k^{*}\right)$ and each element of $\Psi(\hat{k})$ is less than some element of $\Psi(k)$;

(3) if there is a real power product $w$ of elements of $k$ such that $\nu\left(w^{\prime} / w\right) \notin \Psi(k)$ and $\nu\left(w^{\prime} / w\right)$ is the least upper bound of $\Psi(k)$ in $\nu\left(k^{*}\right)$, then

(a) if $\nu(w) \neq 0$, we have $\nu\left(\hat{k}^{*}\right)=\mathbf{R} \nu\left(k^{*}\right), \max \Psi(\hat{k})=\nu\left(w^{\prime} / w\right)=$ l.u.b. $\Psi(k)$, and any other element of $\Psi(\hat{k})$ is less than some element of $\Psi(k)$,

(b) if $\nu(w)=0$ and $w \sim c \in \mathbf{R}^{*}$, then $\mathbf{R} \nu\left(k^{*}\right)+\mathbf{Z} \nu(w-c) \subset \nu\left(\hat{k}^{*}\right) \subset \mathbf{R} \nu\left(k^{*}\right)+$ $\mathbf{R} \nu(w-c)$ and $\max \Psi(\hat{k})=$ l.u.b. $\Psi(k)-\nu(w-c)$, with $\nu(w-c)$ positive and less than any positive element of $\nu\left(k^{*}\right)$, while any other element of $\Psi(\hat{k})$ is less than some element of $\Psi(k)$. These four cases are exhaustive and mutually exclusive.

We already know that $\hat{k}$ is a Hardy field and that $\mathbf{R} \nu\left(k^{*}\right) \subset \nu\left(\hat{k}^{*}\right)$. Recall that for any nonconstant real power product $u$ of elements of $k$ we have $\nu\left(u^{\prime} / u\right) \in \nu\left(k^{*}\right)$. If $\max \Psi(k)$ exists we are in case (1), but not (2) or (3). If $\max \Psi(k)$ does not exist but l.u.b. $\Psi(k)$ exists, we are not in case (1), but we are in precisely one of the two cases (2) or (3). If l.u.b. $\Psi(k)$ does not exist, we are in case (2), but in neither cases (1) nor (3). Thus cases (1), (2), (3) are exhaustive and mutually exclusive. If we are in case (3), then at least one of $(3)(a)$ or (b) must obtain, but not both, as will follow from their different consequences, once these are proved. So it remains to prove the individual consequences of cases (1), (2), (3)(a) and (b). In cases (1) and (2), we use Zorn's lemma to get the existence of a maximal set $U$ of real power products of elements of $k$ such that $k^{*} \subset U$ and $\nu\left((k(U))^{*}\right) \subset \sum_{u \in U} \mathbf{Q} \nu(u)$. Clearly $U$ is a multiplicative group and $k_{1}=k(U)$ is a Hardy subfield of $\hat{k}$, with $\nu\left(k_{1}^{*}\right) \subset \mathbf{Q} \nu(U)$. If $u_{0} \in U$ and $n$ is a positive integer, then $k_{1}\left(u_{0}^{1 / n}\right)$ is an algebraic extension of $k_{1}$, so that $\nu\left(\left(k_{1}\left(u_{0}^{1 / n}\right)\right)^{*}\right) \subset \mathbf{Q} \nu\left(k_{1}^{*}\right) \subset \mathbf{Q} \nu(U)$ and the maximality of $U$ implies that $u_{0}^{1 / n} \in U$. Therefore $\nu\left(k_{1}^{*}\right)=\mathbf{Q} \nu(U)=\nu(U)$. If $z$ is any real power product of elements of $k$ such that $\nu(z) \notin \nu\left(k_{1}^{*}\right)$, then for $a_{0}, a_{1}, \ldots, a_{n} \in k_{1}^{*}$ and not all zero we have

$$
\begin{aligned}
\nu\left(a_{0}+a_{1} z+\cdots+a_{n} z^{n}\right)= & \min \left\{\nu\left(a_{i}\right)+i \nu(z): i=0, \ldots, n\right\} \\
& \in \nu\left(k_{1}^{*}\right)+\mathbf{Z} \nu(z) \subset \sum_{u \in U \cup\{z\}} \mathbf{Q} \nu(u),
\end{aligned}
$$

so that $\nu\left((k(U \cup\{z\}))^{*}\right) \subset \sum_{u \in U \cup\{z\}} \mathbf{Q} \nu(u)$, contradicting the maximality of the set $U$. Thus if $z$ is any real power product of elements of $k$ then there is a $u \in U$ such that $\nu(z)=\nu(u)$. Therefore there is a real power product $w$ of elements of $k$ such that $z / w \in U$ and $w \sim 1$. Looking at the Hardy field $k(w)$, we get $\nu\left(w^{\prime}\right)$ greater than each element of $\Psi(k)$. Also $\nu\left(w^{\prime}\right)=\nu\left(w^{\prime} / w\right) \in \nu\left(k^{*}\right)$. In either case (1) or (2), we can apply Lemma 1 to $\nu\left(w^{\prime}\right)$ to get the existence of an element $t \in k^{*}$ such that $\nu(t)>0$ and $\nu\left(t^{\prime}\right)=\nu\left(w^{\prime}\right)$. If we apply Lemma 2 to $w, t$ and the Hardy fields $k_{1} \subset k_{1}(w)$ we get $\nu\left(\left(k_{1}(w)\right)^{*}\right) \subset \mathbf{Q} \nu\left(k_{1}^{*}\right)$. By the maximality of $U$ we get $w \in U$, so that $z \in U$. In other words, $U$ consists of all real power products of elements of $k$, so that $k_{1}=\hat{k}$ and $\mathbf{R} \nu\left(k^{*}\right) \subset \nu\left(\hat{k}^{*}\right) \subset \nu(U) \subset \mathbf{R} \nu\left(k^{*}\right)$, or $\nu\left(\hat{k}^{*}\right)=\mathbf{R} \nu\left(k^{*}\right)$. 
Therefore

$$
\Psi(\hat{k})=\left\{\nu\left(u^{\prime} / u\right): u \in \hat{k}^{*}, \nu(u) \neq 0\right\}=\left\{\nu\left(u^{\prime} / u\right): u \in U, \nu(u) \neq 0\right\} .
$$

If there were some $u \in U$ such that $\nu(u) \neq 0$ and $\nu\left(u^{\prime} / u\right)$ exceeds each element of $\Psi(k)$ then by Lemma 1 , in either case (1) or (2), there would be an $f \in k^{*}$ such that $\nu(f)>0$ and $\nu\left(f^{\prime}\right)=\nu\left(u^{\prime} / u\right)$, a contradiction. This completes the proof of all our contentions in cases (1) and (2). Now suppose that we are in case (3), with $w$ as indicated in the statement of the theorem. In case (3)(a), Lemma 3 tells us that $k(w)$ is a Hardy field with value group $\nu\left(k^{*}\right)+\mathbf{Z} \nu(w)$, with the comparability class of $w$ smaller than the comparability class of any element of $k$, so that $\Psi(k(w))=\Psi(k) \cup\left\{\nu\left(w^{\prime} / w\right)\right\}$ has a greatest element $\nu\left(w^{\prime} / w\right)$. Applying what has been proved in case (1) to the Hardy field $k(w)$ we get

$$
\nu\left(\hat{k}^{*}\right) \subset \nu\left(\left((k(w))^{\widehat{)}}\right)^{*}\right)=\mathbf{R} \nu\left((k(w))^{*}\right)=\mathbf{R} \nu\left(k^{*}\right)+\mathbf{R} \nu(w)=\mathbf{R} \nu\left(k^{*}\right) \subset \nu\left(\hat{k}^{*}\right),
$$

so that $\nu\left(\hat{k}^{*}\right)=\nu\left(\left((k(w))^{\wedge}\right)^{*}\right)=\mathbf{R} \nu\left(k^{*}\right)$. Since $\nu\left(\hat{k}^{*}\right)=\nu\left(\left((k(w))^{-}\right)^{*}\right)$ we have $\max \Psi(\hat{k})=\max \Psi\left((k(w))^{\hat{\gamma}}\right)=\nu\left(w^{\prime} / w\right)=$ l.u.b. $\Psi(k)$. Since $\Psi(\hat{k})=\left\{\nu\left(u^{\prime} / u\right): u\right.$ a real power product of elements of $k, \nu(u) \neq 0\} \subset \nu\left(k^{*}\right)$, every element of $\Psi(\hat{k})$ other than the maximum l.u.b. $\Psi(k) \notin \Psi(k)$ is less than some element of $\Psi(k)$. This proves everything in case (3)(a). Finally consider case (3)(b). We again use Lemma 3 to get $\nu\left((k(w))^{*}\right)=\nu\left(k^{*}\right)+\mathbf{Z} \nu(w-c)$, with the comparability class of $w-c$ minimal in $k(w)$ and $\nu(w-c)$ less than any positive element of $\nu\left(k^{*}\right)$, so that

$$
\max \Psi(k(w))=\nu\left((w-c)^{\prime} /(w-c)\right)=\text { l.u.b. } \Psi(k)-\nu(w-c) .
$$

Also $k \subset k(w) \subset \hat{k}$. Applying case (1) to $k(w)$, we obtain $\nu\left(\hat{k}^{*}\right) \subset \nu\left(\left((k(w))^{-}\right)^{*}\right)=$ $\mathbf{R} \nu\left((k(w))^{*}\right)=\mathbf{R} \nu\left(k^{*}\right)+\mathbf{R} \nu(w-c)$ and $\max \Psi(\hat{k})=\max \Psi(k(w))$. Any element of $\Psi(\hat{k})$ other than its maximum is of the form $\nu\left(u^{\prime} / u\right)$, with $u$ a real power product of elements of $k$, which is an element of $\nu\left(k^{*}\right)$ less than l.u.b. $\Psi(k)$ and hence less than some element of $\Psi(k)$. This completes the proof of the theorem.

COROLlaRY 1. Notations being as in the theorem,

(1) $\Psi(k) \subset \Psi(\hat{k}) \subset \nu\left(k^{*}\right)$ except in case (3)(b), where $\Psi(k) \subset \Psi(\hat{k}) \subset \nu\left(k^{*}\right) \cup$ $\{\max \Psi(\hat{k})\}$

(2) in cases (1) and (3), $\hat{k}$ satisfies case (1), while, in case (2), $\hat{k}$ satisfies case (2);

(3) in all cases except $(3)(\mathrm{b}), \nu\left(\hat{\hat{k}}^{*}\right)=\nu\left(\hat{k}^{*}\right)$.

Clearly $\Psi(k) \subset \Psi(\hat{k})$. Also, for any $u \in \hat{k}^{*}$ such that $\nu(u) \neq 0$ there is a real power product $u_{1}$ of elements of $k$ (or, in case (3)(b), of elements of $k$ and of $w-c$ ) such that $\nu(u)=\nu\left(u_{1}\right)$, so that $\nu\left(u^{\prime} / u\right)=\nu\left(u_{1}^{\prime} / u_{1}\right)$, which is the value of an element of $k^{*}$ (or of an element of $k^{*}$ plus a real multiple of $(w-c)^{\prime} /(w-c)$ ), so that (1) follows. The first part of (2) is clear. For the second part of (2), suppose that $w$ is a real power product of elements of $\hat{k}$ such that $\nu\left(w^{\prime} / w\right)$ is the least upper bound of $\Psi(\hat{k})$ in $\nu\left(\hat{k}^{*}\right)$. Since $\nu\left(\hat{k}^{*}\right)=\mathbf{R} \nu\left(k^{*}\right)$, there is a real power product $w_{1}$ of elements of $k$ such that $w \sim w_{1}$. If $\nu(w) \neq 0$, then $\nu\left(w^{\prime} / w\right)=\nu\left(w_{1}^{\prime} / w_{1}\right) \in \Psi(\hat{k})$, so that $\nu\left(w^{\prime} / w\right)$ is less than some element of $\Psi(k)$, a contradiction. If $\nu(w)=0$, then for some $c \in \mathbf{R}^{*}$ we have $\nu(w-c)>0$, therefore there is a real power product $v_{1}$ of elements of $k$ such that $v_{1} \sim w-c$, so that $\nu\left(v_{1}^{\prime}\right)=\nu\left(w^{\prime}\right)=\nu\left(w^{\prime} / w\right)=$ l.u.b. $\Psi(\hat{k})$, 
which contradicts the last statement preceding Lemma 1 . So it remains only to verify (3), which is done by noting that a real power product of real power products of certain elements is a real power product of these elements.

COROLlaRY 2. For any Hardy field $k$ that contains $\mathbf{R}$ there is a smallest Hardy field $K \supset k$ such that $|t|^{c} \in \mathbf{R}$ for any $t \in K^{*}$ and any $c \in \mathbf{R}$. Also $\nu\left(K^{*}\right)=\mathbf{R} \nu\left(k^{*}\right)$ or $\nu\left(K^{*}\right)=\mathbf{R} \nu\left(k^{*}\right)+\mathbf{R} \nu(w-c)$, with $w$ a real power product of elements of $k$ and $c \in \mathbf{R}^{*}, \nu(w-c)>0$, and the comparability class of $w-c$ smaller than that of any real power product of elements of $k$, and this last case can occur only if $\Psi(k)$ has a least upper bound in $\nu\left(k^{*}\right)$ that is not in $\Psi(k)$. Furthermore $\operatorname{rank} k \leq \operatorname{rank} K \leq$ rat rank $k$.

Define the sequence of Hardy fields $k_{0}, k_{1}, k_{2}, \ldots$ by setting $k_{0}=k$ and $k_{i+1}=\hat{k}_{i}$ for each $i$. Then $K=\bigcup_{i>0} k_{i}$. Everything is now clear except for the last part, where we reason that

$\operatorname{rank} k \leq \operatorname{rank} K \leq \operatorname{dim}_{\mathbf{R}} \nu\left(K^{*}\right)=\operatorname{dim}_{\mathbf{R}} \mathbf{R} \nu\left(k^{*}\right) \leq \operatorname{dim}_{\mathbf{Q}} \mathbf{Q} \nu\left(k^{*}\right)=\operatorname{rat} \operatorname{rank} k$.

COROLlary 3. Let $k$ be a Hardy field of rank 1 that contains $\mathbf{R}$. Then $\operatorname{rank} \hat{k}=$ $\operatorname{rank} K=1$.

Here we are in case (1) of the theorem, with $\max \Psi(\hat{k})=\max \Psi(K)=\max \Psi(k)$. Therefore if $t \in k^{*}$ is such that $\nu(t) \neq 0$, then $t$ is in the smallest comparability class of $\hat{k}$ or of $K$. On the other hand, it is clear that some integral power of $t$ is ultimately larger than any given element of $\hat{k}$ or of $K$, so that $t$ is also in the largest comparability class of $\hat{k}$ or of $K$.

Because it is so important, it is worth reconsidering the case of a Hardy field $k$ containing $\mathbf{R}$ that is of finite rational rank. Here the earlier proofs simplify considerably, Lemma 1 becoming almost trivial, Lemma 3 unnecessary, and the theorem needing proof only in the relatively easy case (1). The situation may be reviewed in the following suggestive manner. Let $k$ have rational rank $n$, so that there exist $t_{1}, \ldots, t_{n} \in k^{*}$ such that $\nu\left(k^{*}\right) \subset \sum_{i=1}^{n} \mathbf{Q} \nu\left(t_{i}\right)$. Choose a basis $y_{1}, \ldots, y_{m}(m \leq n)$ of the real vector space $S=\sum_{i=1}^{n} \mathbf{R} t_{i}^{\prime} / t_{i} \subset k$ such that $\nu\left(y_{1}\right)=\min \{\nu(y): y \in S\}, \nu\left(y_{2}\right)=\min \left\{\nu(y): y \in S, \nu(y)>\nu\left(y_{1}\right)\right\}, \nu\left(y_{3}\right)=$ $\min \left\{\nu(y): y \in S, \nu(y)>\nu\left(y_{2}\right)\right\}$, etc., so that $\nu\left(y_{1}\right)<\nu\left(y_{2}\right)<\cdots<\nu\left(y_{m}\right)$. For any $u \in k^{*}$ such that $\nu(u) \neq 0$ there exist integers $N, a_{1}, \ldots, a_{n}$, with $N \neq 0$, such that $\nu\left(u^{N}\right)=N \nu(u)=\nu\left(t_{1}^{a_{1}} \cdots t_{n}^{a_{n}}\right)$, so that $\nu\left(u^{\prime} / u\right)=\nu\left(\sum_{i=1}^{n} a_{i} t_{i}^{\prime} / t_{i}\right)$, and therefore $\Psi(k) \subset\left\{\nu\left(y_{i}\right): i=1, \ldots, m\right\}$. If we choose $r$ such that $\max \Psi(k)=\nu\left(y_{r}\right)$, then $r \leq m \leq n$ and the set of all $\nu\left(u^{\prime} / u\right)$ as $u$ ranges over all real power products of elements of $k$ such that $\nu(u) \neq 0$ is precisely the set $\left\{\nu\left(y_{1}\right), \ldots, \nu\left(y_{r}\right)\right\}$. Letting $u_{i}=\exp \left(\int y_{i}\right)$ for $i=1, \ldots, r$, the $u_{i}$ 's are real power products of elements of $k$, each $\nu\left(u_{i}\right) \neq 0$, the comparability classes of $u_{1}, \ldots, u_{r}$ are distinct and in decreasing order, and for any nonzero $u$ in $\hat{k}$ or $K$ we have $\nu(u)=\nu\left(u_{1}^{c_{1}} \cdots u_{r}^{c_{r}}\right)$ for certain unique $c_{1}, \ldots, c_{r} \in \mathbf{R}$. Thus for each $u \in K^{*}$ there is an asymptotic expansion

$$
u \sim c_{1} u_{1}^{c_{11}} \cdots u_{r}^{c_{1 r}}+c_{2} u_{1}^{c_{21}} \cdots u_{r}^{c_{2 r}}+\cdots,
$$

possibly terminating, with each $c_{i}, c_{i j} \in \mathbf{R}$ and each $c_{i}$ nonzero. If each $y_{i}$ had been chosen positive we would have each $u_{i}$ infinitely increasing and the sequence of $r$-tuples $\left\{\left(c_{i 1}, \ldots, c_{i r}\right)\right\}_{i \geq 1}$ would be strictly decreasing in the lexicographical ordering. 


\section{REFERENCES}

1. M. Rosenlicht, Hardy fields, J. Math. Anal. Appl. 93 (1983), 297-311.

2. __ The rank of a Hardy field, Trans. Amer. Math. Soc. 280 (1983), 659-671.

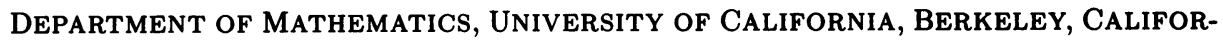
NIA 94720 